\title{
Fuzzy Robust $H \infty$ Tracking Control For Wind Generator System: LMI approach
}

\author{
${ }^{1}$ Kaoutar Lahmadi, ${ }^{2}$ Ismail Boumhidi \\ ${ }^{1,2}$ Department of physics, LESSI Laboratory, Faculty of Sciences Dhar el Mahraz, University Sidi \\ Mohammed Ben Abdellah, Fez, Morocco \\ kaoutar.lahmadi@usmba.ac.ma, iboumhidi@hotmail.com
}

\begin{abstract}
This study concerns the tracking control problem of the wind turbine generator system with uncertainties parameters and external disturbances. Based on T-S fuzzy model, a fuzzy observer-based and a fuzzy robust state feedback output tracking control are developed to reduce the tracking error by minimizing the disturbance level caused by the wind speed. Using a Lyapunov function combined with $\mathrm{H} \infty$ tracking criteria and a judicious of the famous Young relation, a sufficient stability condition for the robust fuzzy tracking control formulated in terms of linear matrix inequality, which can be very efficiently solved by using LMI optimization techniques. The simulation results are given to show the performance of the observer-based tracking controller.
\end{abstract}

Keywords-Wind system, $\mathrm{H} \infty$ tracking control, observer-based controller, linear matrix inequality (LMI).

\section{Introduction}

The Takagi -Sugeno (T-S) fuzzy model approach has nowadays become popular since [1][2][6], it showed its efficiency to control complex nonlinear systems with uncertainties parameters and used for many applications [7]. This fuzzy dynamic model is a piecewise interpolation of several linear models through membership functions [4]. It is described by fuzzy rules of the type IF-THEN that represent local input output models for a nonlinear system [2]. For more details on these topics we refer the reader to Tanaka and Wang [6].

For a few years, the trend of fuzzy tracking control for uncertain nonlinear systems has attracted great attention [14] [8]. The main issue in the control theory is that of controlling a system in order to have its output asymptotically tracking reference signals [10]. Despite an abundant literature on stability conditions of T-S models, few authors have dealt with the tracking problem. Some works are concerned with state feedback, $\mathrm{H}_{\infty}$ performances and output feedback [12]. The most important issue for fuzzy tracking control systems is how to reduce the tracking error between the desired trajectory and the actual output values rapidly with the guaranteed stability [16]. In this work a sufficient condition are derived for stabilization of the robust fuzzy tracking controller and the robust fuzzy observer in the sense of Lyapunov asymptotic stability [15]. 
Kaoutar Lahmadi, Ismail Boumhidi; Fuzzy Robust Hoo Tracking Control For Wind Generator System: LMI approach. Transactions on Machine Learning and Artificial Intelligence, Vol 5 No 4 August (2017); pp: 43-54

In the last decade, optimal $\mathrm{H} \infty$ control theory has been well developed and found extensive application to efficiently treat the robust stabilization and disturbance rejection problems. The $\mathrm{H} \infty$ control performance for uncertain nonlinear systems is proposed to attenuate the effects caused by modeled dynamics, disturbances and approximate errors [13].

The task of tracking is a typical control problem in the industry. In this study we focused in control of wind system formed by a turbine, a driving shaft and a double fed induction machine .As well known, the primary energy source of the wind generators is the wind which is unpredictable and varies frequently [9]. Wind energy has greatly progressed by becoming one of most renewable energy competitive [5].The control plays a very important role to make wind technology more profitable and reliable. Then an $\mathrm{H}_{\infty}$ output feedback controller is designed by taking into account directly wind speed as perturbation [15].

In this paper, the tracking problem for wind turbine is studied using the T-S fuzzy approach. First a fuzzy augmented system is constructed by regrouping the T-S fuzzy model, a fuzzy observer, a fuzzy tracking control. Then, based on Lyapunov function combined with a Young Relation and $\mathrm{H} \infty$ criteria a new condition for stabilization of the whole system is obtained. This condition is applied on wind turbine system with uncertainties parameters and external disturbances in order to guarantying the performances and to reduce the tracking error by minimizing the disturbance level caused by the wind speed. The proposed methodology is formulated as the linear matrix inequality problem (LMIs) which can be solved very efficiently using the convex optimization techniques.

The paper is arranged as follows: the uncertain T-S fuzzy model with external disturbances, the tracking criteria as well as the observer design and the tracking control law are described in section (II). In section (III) a sufficient condition based on the observer-based tracking control and using Lyapunov function is developed in order to in to minimize the tracking error. Section (IV) presents the performances of the proposed method according to robustness tests are shown through simulations of the wind turbine in. Section (V) concludes this paper.

\section{Problem formulation}

\subsection{T-S fuzzy model}

Let consider the T-S fuzzy system with uncertainties parameters. The i-th rule is described as follows: Plant rule:

If $\quad z_{1}(t)$ is $N_{i j}$ and $\ldots$ and $z_{p}(t)$ is $N_{i p}$

Then

$$
\left\{\begin{array}{l}
\dot{x}(t)=\left(A_{i}+\Delta A_{i}\right) x(t)+\left(B_{i}+\Delta B_{i}\right) u(t)+B_{2 i} \varphi(t) \\
y(t)=C_{i} x(t)
\end{array}\right.
$$

Where $N_{i j}$ a fuzzy set is, $x(t) \in R^{n}$ is the system state vector, $u(t) \in R^{m}$ is the control input vector, $\varphi(t) \in R^{p}$ is the disturbance input vector, $y(t) \in R^{p}$ is the system output, $A_{i}, B_{i}, C_{i}$ are known constant matrix that describe the nominal system, $z_{1}(t)=\left[z_{1}(t), z_{2}(t), \ldots \ldots . ., z_{p}(t)\right]$ are the premise variables. The Lebesgue measurable uncertainties are defined as 
$\Delta A_{i}(t)=H_{i} F_{i}(t) E_{a i}, \Delta B_{i}(t)=H_{i} F_{i}(t) E_{b i}$, where matrices $H_{i}, E_{a i}$ and $E_{b i}$ are constant of appropriate dimension and $F(t)$ is unknown matrix function which is bounded by: $F_{i}{ }^{T}(t) F_{i}(t) \leq I$

Given a pair of $(x(t), u(t))$, the final outputs of the fuzzy systems are inferred as follows:

$$
\dot{x}(t)=\frac{\sum_{i=1}^{r} \mu_{i}(z(t))\left[\left(A_{i}+\Delta A_{i}(t)\right) x(t)+\left(B_{i}+\Delta B_{i}(t)\right) u(t)+B_{2 i} \omega(t)\right]}{\sum_{i=1}^{r} \mu_{i}(z(t))}
$$

Where $\mu_{i}=\prod_{i=1}^{n} N_{i j} z(t)$ and $\quad h_{i}(z(t))=\frac{\mu_{i}(z(t))}{\sum_{i=1}^{r} \mu_{i}(z(t))}$

The defuzzification process of the TS fuzzy model (1) with uncertainties parameters can be represented as:

$$
\left\{\begin{array}{l}
\dot{x}(t)=\sum_{=1}^{*} h_{i}(z(t))\left[\left(A_{i}+\Delta A_{i}(t)\right) x(t)+\left(B_{i}+\Delta B_{i}(t)\right) u(t)+B_{2 i} \varphi(t)\right] \\
y(t)=\sum_{i=1}^{r} h_{i}(z(t)) C_{i} x(t)
\end{array}\right.
$$

$i=1,2 \ldots \ldots ., r$

\subsection{Observer design}

In the present work, the case where the state variables are not available for the feedback control with unmeasurable premise variable is considered.Therfore, the following T-S observer is considered to deal with the state estimation of T-S nonlinear system (2).

$$
\left\{\begin{array}{l}
\dot{\hat{x}}(t)=\sum_{i=1}^{r} A_{i} \hat{x}(t)+B_{i} u(t)+G_{i}(y(t)-\hat{y}(t)) \\
\hat{y}(t)=\sum_{i=1}^{r} C_{i} \hat{x}(t)
\end{array}\right.
$$

Where $G_{i}(i=1, \ldots, r)$ are the observers gains to be determined and $\hat{x}(t)$ is the state estimation.

Let us define the state estimation error $e_{x}(t)$ as:

$$
e_{x}(t)=x(t)-\hat{x}(t) \dot{e}_{x}(t)=\dot{x}(t)-\dot{\hat{x}}(t)
$$

\subsection{Tracking criteria}

To deal with the tracking problem for the T-S fuzzy system, we consider the following reference model:

$$
\dot{x}_{r}(t)=A_{r}(t) x_{r}(t)+r(t)
$$

$x_{r}(t)$ the reference state;

$A_{r}(t)$ a specified asymptotically stable matrix;

$r(t)$ a bounded reference input.

Then the objective is to design a T-S fuzzy model-based controller, which stabilizes the fuzzy system (2) and achieves the $H_{\infty}$ tracking performance related to tracking error $e_{r}(t)$ as follows [tong 2002]:

$$
\int_{0}^{t_{f}} e_{r}^{T}(t) Q e_{r}(t) d(t) \leq \eta^{2} \int_{0}^{t_{f}} \bar{\phi}^{T}(t) \bar{\phi}(t) d(t)
$$


Where $t_{f}$ denotes the final time, $Q$ is a positive definite weighting matrix, and $\eta$ is a specified attenuation level.

The tracking error is defined by:

$$
e_{r}(t)=x(t)-x_{r}(t) \dot{e}_{r}(t)=\dot{x}(t)-\dot{x}_{r}(t)
$$

\subsection{Tracking control:}

Suppose the following fuzzy controller is employed to deal with the above control system design, the control structure is choosen as a PDC law:

$$
u(t)=-\sum_{i=1}^{r} h_{i}(z(t)) k_{i}\left(x_{r}(t)-\hat{x}(t)\right)
$$

Where $k_{i}$ are gain matrices with appropriate dimension.

Let us consider the estimation error $e_{x}(t)=x(t)-\hat{x}(t)$, the tracking error state reference $e_{r}(t)=x(t)-x_{r}(t)$ and the state reference $x_{r}(t)$. By substituting (2),(3) and (8)in (4), and substituting (2), (5)and (8) in (7) we get :

$$
\begin{gathered}
\dot{e}_{x}(t)=\sum_{i=1}^{r} h_{i}(z(t))\left[\left(A_{i}-L_{i} C_{i}\right) e_{x}(t)+\Delta A(t) e_{r}(t)\right. \\
\left.+\Delta A(t) x_{r}(t)+\Delta B(t) k_{j}\left(e_{r}(t)-e_{x}(t)\right)+B_{2 i} \omega(t)\right] \\
\dot{e}_{r}(t)=\sum_{i=1}^{r} h_{i}(z(t))\left[\left(A_{i}-A_{r}+\Delta A(t)\right) x_{r}(t)+\left(A_{i}+\Delta A(t)\right) e_{r}(t)\right. \\
\left.+\left(B_{i}+\Delta B(t)\right) k_{j}\left(e_{r}(t)-e_{x}(t)\right)+B_{2 i} \omega(t)-r(t)\right] \\
\dot{x}_{r}(t)=A_{r}(t) x_{r}(t)+r(t)
\end{gathered}
$$

The state vector for the global closed loop is $x_{a}^{T}(t)=\left(\begin{array}{llll}e_{r}^{T}(t) & e_{x}^{T}(t) & \left.x_{r}^{T}(t)\right)\end{array}\right.$

Some easy manipulations lead to the following augmented system:

$$
\dot{x}_{a}(t)=\sum_{i=1}^{r} \sum_{j=1}^{r} h_{i}(z(t)) h_{j}(z(t))\left(\bar{A}_{i j}(t) x_{a}(t)+\bar{S} \bar{\phi}(t)\right)
$$

Where:

$$
\begin{gathered}
\bar{\phi}(t)=\left[\begin{array}{c}
r(t) \\
\varphi(t)
\end{array}\right] \\
\bar{S}(t)=\left[\begin{array}{cc}
-I & B_{2 i} \\
0 & B_{2 i} \\
I & 0
\end{array}\right] \\
\bar{A}_{j j}(t)=\left[\begin{array}{ccc}
A_{i}+\Delta A_{i}(t)+B_{i} k_{j}+\Delta B_{i}(t) k_{j} & -B_{i} k_{j}-\Delta B_{i}(t) k_{j} & A_{i}-A_{r}+\Delta A_{i}(t) \\
\Delta A_{i}(t)+\Delta B_{i}(t) k_{j} & A_{i}-L_{j} C_{i}-\Delta B_{i}(t) k_{j} & \Delta A_{i}(t) \\
0 & 0 & A_{r}
\end{array}\right]
\end{gathered}
$$

Hence, the tracking criteria $H_{\infty}(6)$ with the augmented vector $x_{a}(t)$ can be modified as follows:

$$
\int_{0}^{t f} x_{a}^{T}(t) Q_{a} x_{a}(t) d(t) \leq \eta^{2} \int_{0}^{t f} \bar{\phi}^{T}(t) \bar{\phi}(t) d(t)
$$

With $Q_{a}=(Q, 0,0,0)$ and $\bar{\phi}^{T}(t) \bar{\phi}(t)=r^{T}(t) r(t)+\varphi^{T}(t) \varphi(t)$ 


\section{LMI Formulation of the Fuzzy Observer-Based Tracking Control}

The design purpose in this study is to determine the gains $k_{j}$ and $L_{j}$ described in (9) to ensure the asymptotic stability of the closed loop system (9) and achieve the $H_{\infty}$ tracking performance in (10). The main result on the fuzzy tracking control design of T-S fuzzy model with uncertainties parameters are summarized in the following theorem:

Theorem .Given the uncertain system described by (2).If there exist a symmetric and positive definite $P=P^{T} \succ 0$ and $Q \succ 0$ some matrices $X_{j}, W_{j}$ and a positive scalars $\eta, \mu_{1}, \mu_{2}, \mu_{3}$ and $\mu_{4}$, such that the following condition (11) hold for $i, j=(1, \ldots, r)$ :

$$
\left[\begin{array}{cccccccccc}
\Sigma_{11} & N & -B_{i} X_{j} & \Sigma_{14} & N^{T} E_{a i}^{T} & N^{T} E_{a i}^{T} & X_{j}^{T} E_{b i}^{T} & -X_{j}^{T} E_{b i}^{T} & -N & B_{2 i} N \\
* & -Q^{-1} & 0 & 0 & 0 & 0 & 0 & 0 & 0 & 0 \\
* & * & \Sigma_{33} & 0 & 0 & 0 & 0 & 0 & 0 & P_{2} B_{2 i} \\
* & * & * & \Sigma_{44} & 0 & 0 & 0 & 0 & P_{3} & 0 \\
* & * & * & * & -\mu_{1}^{-1} I_{1} & 0 & 0 & 0 & 0 & 0 \\
* & * & * & * & * & -\mu_{2}^{-1} I_{2} & 0 & 0 & 0 & 0 \\
* & * & * & * & * & * & -\mu_{3}^{-1} I_{3} & 0 & 0 & 0 \\
* & * & * & * & * & * & * & -\mu_{4}^{-1} I_{4} & 0 & 0 \\
* & * & * & * & * & * & * & * & -\eta^{2} I_{5} & 0 \\
* & * & * & * & * & * & * & * & * & -\eta^{2} I_{6}
\end{array}\right] \prec 0
$$

With:

$\Sigma_{11}=N_{i} A_{i}^{T}+A_{i} N+X_{j}^{T} B_{i}^{T}+B_{i} X_{j}+\left(\mu_{1}^{-1}+\mu_{2}^{-1}+\mu_{3}^{-1}+\mu_{4}^{-1}\right) H_{i} H_{i}^{T}$

$\Sigma_{14}=A_{i} N-A_{r} N$

$\Sigma_{33}=A_{i}^{T} P_{2}+P_{2} A_{i}-W_{j}^{T} C_{i}^{T}-C_{i} W_{j}$

$\Sigma_{44}=A_{r}^{T} P_{3}+P_{3} A_{r}$

The controller and observer gains are given $j=\left(1, \ldots .\right.$, by $\begin{array}{l}N=P_{1}^{-1} \\ X_{j}=k_{j} P_{1}^{-1}\end{array}$

$$
W_{j}=P_{2} L_{j}
$$

Proof. Let us now consider the following candidate Lyapunov function for the augmented system (9):

$$
V(t)=x_{a}^{T}(t) P x_{a}(t)
$$

Where the weighting matrix $P=P^{T} \succ 0$

Using (9) the time derivative of $V(t)$ is the following by

$$
\dot{V}(t)=\dot{x}_{a}^{T}(t) P x_{a}(t)+x_{a}^{T}(t) P \dot{x}_{a}(t)
$$

Substituting (9) into (13) we get:

$$
\begin{aligned}
\dot{V}(t)= & \sum_{i=1}^{r} \sum_{j=1}^{r} h_{i}(z(t)) h_{j}(z(t))\left[x_{a}^{T}(t)\left(\bar{A}_{i j}^{T}(t) P+P^{T} \bar{A}_{i j}(t)\right) x_{a}(t)\right. \\
& \left.+\bar{\phi}^{T}(t) \bar{S}^{T} P x_{a}(t)+x_{a}^{T}(t) P^{T} \bar{S} \bar{\phi}(t)\right]
\end{aligned}
$$

To ensure the stability of the augmented system (9) and guarantying the $H_{\infty}$ performances in (10), the condition to verify is:

$$
\dot{V}(t)+x_{a}^{T}(t) Q_{a} x_{a}(t)-\eta^{2} \bar{\phi}^{T}(t) \bar{\phi}(t) \prec 0
$$


From equations (14) and (9), (15) becomes: $\sum_{i=1}^{r} h_{i}(z(t)) \sum_{j=1}^{r} h_{j}(z(t))\left[x_{a}^{T}(t) \quad \bar{\phi}^{T}(t)\right]$

$$
\left[\begin{array}{cc}
\bar{A}_{i j}^{T} P+P \bar{A}_{i j}+Q_{a} & P \bar{S} \\
\bar{S}^{T} P & -\eta^{2} I
\end{array}\right]\left[\begin{array}{c}
x_{a}(t) \\
\bar{\phi}(t)
\end{array}\right] \prec 0
$$

This is satisfied if:

$$
\left[\begin{array}{cc}
\bar{A}_{i j}^{T} P+P \bar{A}_{i j}+Q_{a} & P \bar{S} \\
\bar{S}^{T} P & -\eta^{2} I
\end{array}\right] \prec 0
$$

Fortunately (16) can be transferred into a LMI problem which can be solved by using convex optimization techniques. Let us assume that $P=\operatorname{diag}\left(P_{1}, P_{2}, P_{3}\right)$ with $P_{1}=P_{1}^{T} \succ 0, P_{2}=P_{2}^{T} \succ 0, P_{3}=P_{3}^{T} \succ 0$

Substitution of $P$ into (16) yields:

$$
\left[\begin{array}{c}
e_{r}^{T}(t) \\
e_{x}^{T}(t) \\
x_{r}^{T}(t) \\
r^{T}(t) \\
\varphi^{T}(t)
\end{array}\right]^{T}\left[\begin{array}{ccccc}
Y_{11} & Y_{12} & Y_{13} & -P_{1} & P_{1} B_{2 i} \\
* & Y_{22} & Y_{23} & 0 & P_{2} B_{2 i} \\
* & * & Y_{33} & P_{3} & 0 \\
* & * & * & -\eta^{2} I & 0 \\
* & * & * & * & -\eta^{2} I
\end{array}\right]\left[\begin{array}{c}
e_{r}(t) \\
e_{x}(t) \\
x_{r}(t) \\
r(t) \\
\varphi(t)
\end{array}\right] \prec 0
$$

With:

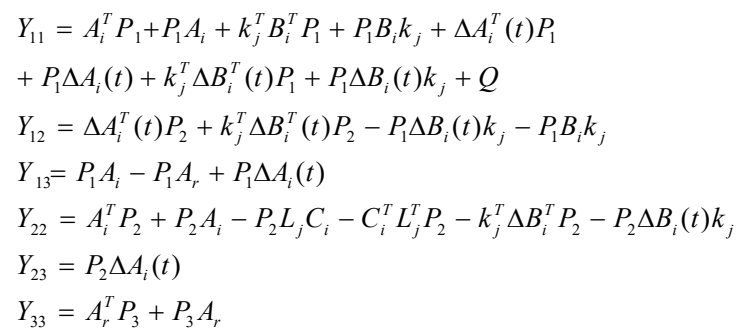

In order to solve the matrix inequality above we rewrite the matrix (18) in format of to matrices the constant term and the uncertainties term as follows:

$$
\left[\begin{array}{ccccc}
A_{11} & A_{12} & A_{13} & -P_{1} & P_{1} B_{2 i} \\
* & A_{22} & 0 & 0 & P_{2} B_{2 i} \\
* & * & A_{33} & P_{3} & 0 \\
* & * & * & -\eta^{2} I & 0 \\
* & * & * & * & -\eta^{2} I
\end{array}\right]+\left[\begin{array}{ccccc}
B_{11} & B_{12} & B_{13} & 0 & 0 \\
* & B_{22} & B_{23} & 0 & 0 \\
* & * & 0 & 0 & 0 \\
* & * & * & 0 & 0 \\
* & * & * & * & 0
\end{array}\right] \prec 0 \quad \text { With: }
$$

$$
\begin{aligned}
& A_{11}=A_{i}^{T} P_{1}+P_{1} A_{i}+k_{j}^{T} B_{i}^{T} P_{1}+P_{1} B_{i} k_{j}+Q \\
& A_{12}=-P_{1} B_{i} k_{j} \\
& A_{13}=P_{1} A_{i}-P_{1} A_{r} \\
& A_{22}=A_{i}^{T} P_{2}+P_{2} A_{i}-P_{2} L_{j} C_{i}-C_{i}^{T} L_{j}^{T} P_{2} \\
& A_{33}=A_{r}^{T} P_{3}+P_{3} A_{r} \\
& B_{11}=\Delta A_{i}^{T}(t) P_{1}+P_{1} \Delta A_{i}(t)+k_{j}^{T} \Delta B_{i}^{T}(t) P_{1}+P_{1} \Delta B_{i}(t) k_{j} \\
& B_{12}=\Delta A_{i}^{T}(t) P_{2}+k_{j}^{T} \Delta B_{i}^{T}(t) P_{2}-P_{1} \Delta B_{i}(t) k_{j} \\
& B_{13}=P_{1} \Delta A_{i}(t) \\
& B_{22}=-k_{j}^{T} \Delta B_{i}^{T} P_{2}-P_{2} \Delta B_{i}(t) k_{j} \\
& B_{23}=P_{2} \Delta A_{i}(t)
\end{aligned}
$$


By introducing new variables $N=P^{-1}, X_{j}=k_{j} P_{1}^{-1}$ and $W_{j}=P_{2} L_{j}$ after per-post-multiply the inequality (19) by $\operatorname{diag}\left[P_{1}^{-1} \quad I \quad I \quad I \quad I\right]$ we obtain the following form:

$$
\left[\begin{array}{ccccc}
\bar{A}_{11} & \bar{A}_{12} & \bar{A}_{13} & -N_{1} & B_{2 i} N \\
* & \bar{A}_{22} & 0 & 0 & P_{2} B_{2 i} \\
* & * & \bar{A}_{33} & P_{3} & 0 \\
* & * & * & -\eta^{2} I & 0 \\
* & * & * & * & -\eta^{2} I
\end{array}\right]+\left[\begin{array}{ccccc}
\bar{B}_{11} & \bar{B}_{12} & \bar{B}_{13} & 0 & 0 \\
* & \bar{B}_{22} & \bar{B}_{23} & 0 & 0 \\
* & * & 0 & 0 & 0 \\
* & * & * & 0 & 0 \\
* & * & * & * & 0
\end{array}\right] \prec 0
$$

$$
\begin{aligned}
& \bar{A}_{11}=N^{T} A_{i}^{T}+A_{i} N+X_{j}^{T} B_{i}^{T}+B_{i} X_{j}+N Q N \\
& \bar{A}_{12}=-B_{i} X_{j} \\
& \bar{A}_{13}=A_{i} N-A_{r} N \\
& \bar{A}_{22}=A_{i}^{T} P_{2}+P_{2} A_{i}-W_{j} C_{i}-C_{i}^{T} W_{j}^{T} \\
& \bar{A}_{33}=A_{r}^{T} P_{3}+P_{3} A_{r} \\
& \bar{B}_{11}=N^{T} \Delta A_{i}^{T}(t)+\Delta A_{i}(t) N+X_{j}^{T} \Delta B_{i}^{T}(t)+\Delta B_{i}(t) X_{j} \\
& \bar{B}_{12}=N^{T} \Delta A_{i}^{T}(t)+X_{j}^{T} \Delta B_{i}^{T}(t)-\Delta B_{i}(t) X_{j} \\
& \bar{B}_{13}=\Delta A_{i}(t) N \\
& \bar{B}_{22}=-X_{j}^{T} \Delta B_{i}^{T}-\Delta B_{i}(t) X_{j} \\
& \bar{B}_{23}=\Delta A_{i}(t) N
\end{aligned}
$$

Then using the uncertainties structure defined in (1) the term with uncertainties in (20) becomes:

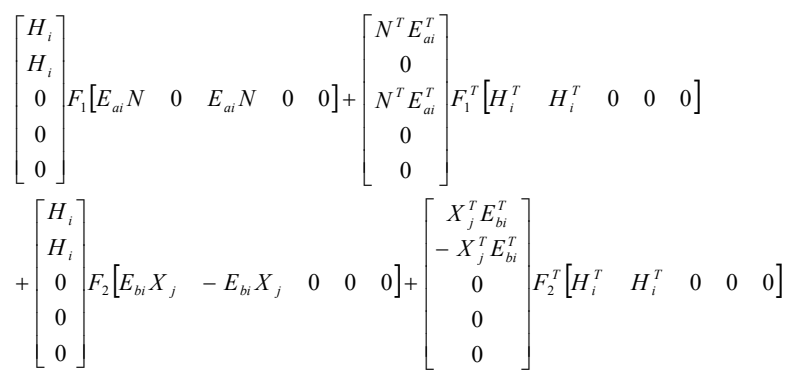

The following lemmas are needed to propose LMI conditions:

Lemma 1.[] Given constant matrices $H_{i}$ and $E_{a i}, E_{b i}$ of appropriate dimensions , $\mu_{i} \succ 0$ and $F(t)$ satisfied $F^{T}(t) F(t) \leq I$ the following inequality holds :

$$
H_{i} F(t) E_{a i}+E_{a i}^{T} F^{T}(t) H_{i}^{T} \leq \mu_{i}^{-1} H_{i} H_{i}^{T}+\mu_{i} E_{a i}^{T} E_{a i}
$$

Lemma 2. [] shur complement the LMI:

$$
\left[\begin{array}{cc}
Q(y)+\rho . I & S(y) \\
S^{T}(y) & R(y)
\end{array}\right] \prec 0
$$

is equivalent to $R(y) \prec 0, Q(y)-S(y) R(y)^{-1} S^{T}(y) \prec-\rho . I \quad$ where $Q(y)=Q^{T}(y), R(y)=R^{T}(y) \quad$ and $S(y)$ depend affinely on $y$ and $\rho$ is a real number.

Remark 1. The parameter uncertainties $\Delta A_{i}(t), \Delta B_{i}(t)$ will represent the impossibility for exact mathematical model of a dynamic system due to the system complexity. The uncertainty has been widely used in many practical systems [11] [12].

By applying lemma .1 in term with uncertainties (21) we have: 


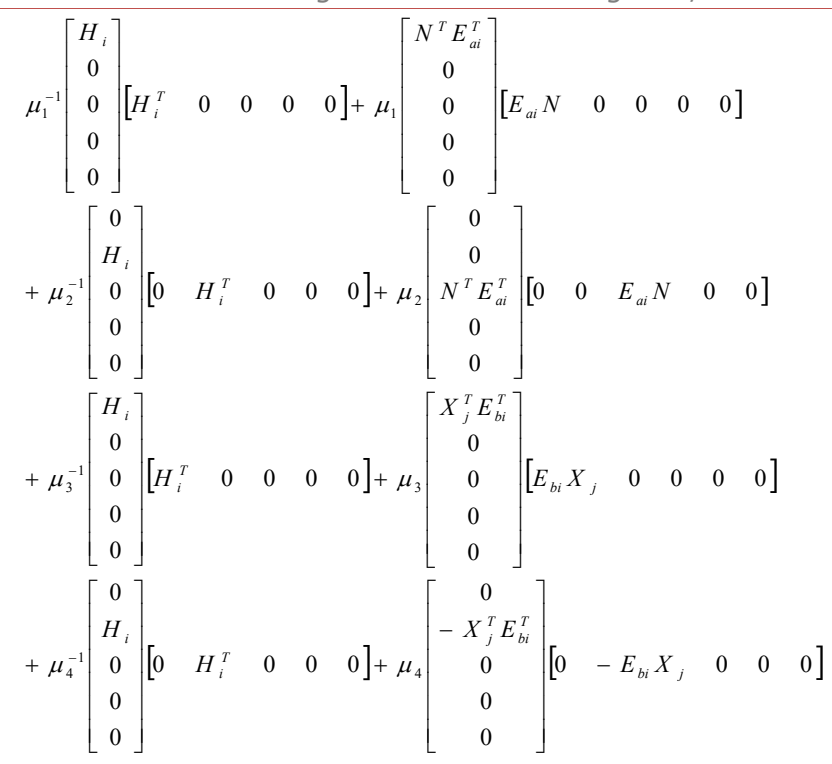

Then, replacing (22) and (20) in (19) and applying lemma.2, we get the LMI (11), this condition can be efficiently solved numerically by using a linear matrix inequality (LMI) framework, wich ends the proof.

\section{Example and Simulation}

To illustrate the proposed fuzzy robust tracking control condition, a control problem of a wind generator is considered.

By defining the state vector $x(t)=\left[\begin{array}{llll}\theta_{s} & \Omega_{r} & \Omega_{g} & \beta\end{array}\right]^{T}$ and the control signal as $u=\left[\begin{array}{ll}\beta_{d}, \Omega_{z}\end{array}\right]^{T}$, The reference state vector to track is defined as $x_{r}(t)=\left[\begin{array}{llll}\theta_{r s} & \Omega_{r r} & \Omega_{g r} & \beta_{r}\end{array}\right]^{T}$ the fuzzy model of the WECS can be described as:

$$
\left\{\begin{array}{l}
\dot{x}(t)=A(z) x(t)+B u(t)+B_{2} V(t) \\
y(t)=C x(t)
\end{array}\right.
$$

Where:

$$
\begin{gathered}
A(z)=\left[\begin{array}{cccc}
0 & 1 & -1 & 0 \\
-\frac{K_{s}}{J_{r}} & -\frac{B_{s}}{J_{r}} & \frac{B_{s}}{J_{r}} & \frac{T_{r \beta}\left(z_{0}\right)}{J_{r}} \\
\frac{K_{s}}{J_{g}} & \frac{B_{s}}{J_{g}} & -\frac{\left(B_{s}+B_{g}\right)}{J_{g}} & 0 \\
0 & 0 & 0 & -\frac{1}{\tau}
\end{array}\right] \\
B=\left[\begin{array}{cc}
0 & 0 \\
0 & 0 \\
0 & \frac{B_{g}}{J_{g}} \\
\frac{1}{\tau} & 0
\end{array}\right] \quad B_{2}=\left[\begin{array}{c}
0 \\
\frac{T_{r v}\left(z_{0}\right)}{J_{r}} \\
0 \\
0
\end{array}\right]
\end{gathered}
$$

Where $\theta_{s}$ denotes the torsion angle, $\Omega_{r}$ is angular velocity of rotor, $\Omega_{g}$ is angular velocity of generator, $K_{s}$ is the stiffness of the transmission, $B_{s}$ is the damping of the transmission, $J_{r}$ and $J_{g}$ are the inertia of the rotor and generator, respectively. 
$T_{r}$ is the aerodynamic torque. $\beta$ and $\beta_{d}$ are the actual and desired pitch angles, respectively.

\subsection{T-S Fuzzy Modeling:}

The wind generator system is then described by the following four IF-THEN rules:

If $\beta$ is $F_{1}^{1}$ and $V$ is $F_{2}^{1}$ then $\left\{\begin{array}{l}\dot{x}=A_{1} x+B_{1} u+B_{21} \varphi \\ y=C x\end{array}\right.$

If $\beta$ is $F_{1}^{1}$ and $V$ is $F_{2}^{2}$ then $\left\{\begin{array}{l}\dot{x}=A_{2} x+B_{2} u+B_{22} \varphi \\ y=C x\end{array}\right.$

If $\beta$ is $F_{1}^{2}$ and $V$ is $F_{2}^{1}$ then $\left\{\begin{array}{l}\dot{x}=A_{3} x+B_{3} u+B_{23} \varphi \\ y=C x\end{array}\right.$

If $\beta$ is $F_{1}^{2}$ and $V$ is $F_{2}^{2}$ then $\left\{\begin{array}{l}\dot{x}=A_{4} x+B_{4} u+B_{24} \varphi \\ y=C x\end{array}\right.$

In order to obtain the best performance from this non linear system (23) the following T-S fuzzy model is given:

$$
\left\{\begin{array}{l}
\dot{x}(t)=\sum_{i=1}^{4} h_{i}(z(t))\left[\left(A_{i}+\Delta A_{i}(t)\right) x(t)+\left(B_{i}+\Delta B_{i}(t)\right) u(t)+B_{2 i} \varphi(t)\right] \\
y(t)=\sum_{i=1}^{4} h_{i}(z(t)) C_{i} x(t)
\end{array}\right.
$$

The parameter uncertainties $\Delta A_{i}(t), \Delta B_{i}(t)$ represent the impossibility for exact mathematical model of a dynamic system due to the system complexity. The wind generator is a complex system then the presence of uncertainties is possible.

Where:

$$
\begin{aligned}
& \varphi(t)=V(t) \\
& h_{1}(z)=F_{1}^{1}(\beta) F_{2}^{1}(V), \quad h_{1}(z)=F_{1}^{1}(\beta) F_{2}^{1}(V) \text {, } \\
& h_{2}(z)=F_{1}^{1}(\beta) F_{2}^{2}(V) \quad h_{2}(z)=F_{1}^{1}(\beta) F_{2}^{2}(V) \\
& h_{3}(z)=F_{1}^{2}(\beta) F_{2}^{1}(V), \quad h_{3}(z)=F_{1}^{2}(\beta) F_{2}^{1}(V) \text {, } \\
& h_{4}(z)=F_{1}^{2}(\beta) F_{2}^{2}(V) \quad h_{4}(z)=F_{1}^{2}(\beta) F_{2}^{2}(V) \\
& A_{1}=\left[\begin{array}{cccc}
0 & 1 & -1 & 0 \\
-\frac{K_{s}}{J_{r}} & -\frac{B_{s}}{J_{r}} & \frac{B_{s}}{J_{r}} & \frac{T_{r \beta 1}}{J_{r}} \\
\frac{K_{s}}{J_{g}} & \frac{B_{s}}{J_{g}} & -\frac{\left(B_{s}+B_{g}\right)}{J_{g}} & 0 \\
0 & 0 & 0 & -\frac{1}{\tau}
\end{array}\right] \\
& A_{3}=\left[\begin{array}{cccc}
0 & 1 & -1 & 0 \\
-\frac{K_{s}}{J_{r}} & -\frac{B_{s}}{J_{r}} & \frac{B_{s}}{J_{r}} & \frac{T_{r \beta 2}}{J_{r}} \\
\frac{K_{s}}{J_{g}} & \frac{B_{s}}{J_{g}} & -\frac{\left(B_{s}+B_{g}\right)}{J_{g}} & 0 \\
0 & 0 & 0 & -\frac{1}{\tau}
\end{array}\right]
\end{aligned}
$$




$$
\begin{gathered}
A_{2}=A_{1}, A_{4}=A_{3} \\
B=\left[\begin{array}{cc}
0 & 0 \\
0 & 0 \\
0 & \frac{B_{g_{g}}}{J_{g}} \\
\frac{1}{\tau} & 0
\end{array}\right] B_{21}=B_{23}=\left[\begin{array}{c}
0 \\
\frac{T_{r v 1}}{J_{r}} \\
0 \\
0
\end{array}\right] B_{22}=B_{24}=\left[\begin{array}{c}
0 \\
\frac{T_{r v 2}}{J_{r}} \\
0 \\
0
\end{array}\right]
\end{gathered}
$$

$C=\left[\begin{array}{llll}0 & 0 & 1 & 0\end{array}\right]$

$B=B_{1}=B_{2}=B_{3}=B_{4}$

$C=C_{1}=C_{2}=C_{3}=C_{4}$

Numerical value:

$K_{s}=1.566 * 10{ }^{6} \mathrm{~N} / \mathrm{m}, \tau=100 \mathrm{~ms}$

$B_{s}=3029.5 \mathrm{Nms} / \mathrm{rad}, B_{g}=15.993 \mathrm{Nms} / \mathrm{rad}$

$J_{r}=830000 \mathrm{Kg} \cdot \mathrm{m}^{2}, J_{g}=5.9 \mathrm{Kg} \cdot \mathrm{m}^{2}$

$T_{r \beta 1}=723980 T_{r \beta 2}=37607 \mathrm{C}$

$T_{r v 1}=10644 Q T_{r v 2}=85370$

The control objective to attain is the best tracking of rated power while regulating rotor speed.

We consider output tracking control network-based T-S fuzzy system described by (24), we show the effectiveness of the proposed method by performing output tracking control for the T-S fuzzy system.

Solving LMI (11) by using the Toolbox LMI optimization algorithm, feedback and observer gain matrices can be obtained as:

$$
\begin{aligned}
& k_{1}=\left[\begin{array}{rrrr}
-0.0000 & -0.0000 & 0.0000 & 0.0000 \\
-0.0003 & -0.0000 & -0.0457 & -0.0000
\end{array}\right] \\
& k_{2}=\left[\begin{array}{rrrr}
-0.0000 & -0.0000 & 0.0000 & 0.0000 \\
-0.0003 & -0.0000 & -0.0457 & -0.0000
\end{array}\right] \\
& k_{3}=\left[\begin{array}{rrrr}
-0.0000 & -0.0000 & 0.0000 & 0.0000 \\
-0.0003 & -0.0000 & -0.0457 & -0.0000
\end{array}\right] \\
& k_{4}=\left[\begin{array}{rrrr}
-0.0000 & 0.0000 & 0.0000 & -0.0000 \\
-0.0003 & -0.0000 & -0.0568 & -0.0000
\end{array}\right] \\
& L_{1}=\left[\begin{array}{l}
0.0003 \\
0.0000 \\
0.0764 \\
0.0000
\end{array}\right], L_{2}=\left[\begin{array}{l}
0.0003 \\
0.0000 \\
0.0764 \\
0.0000
\end{array}\right] \\
& L_{3}=\left[\begin{array}{l}
0.0003 \\
0.0000 \\
0.0764 \\
0.0000
\end{array}\right], L_{4}=\left[\begin{array}{l}
0.0004 \\
0.0000 \\
0.0877 \\
0.0000
\end{array}\right]
\end{aligned}
$$

The simulations results of LMI (11) show that the attenuation level is $\eta=0.3442$ so, we can deduce that the designed T-S fuzzy controller ensures a good tracking performance and can to guarantee the stability.

For simulation, we consider the wind speed input profile 
$(17 \mathrm{~m} / \mathrm{s}<\mathrm{V}<31 \mathrm{~m} / \mathrm{s})$ as given in Figure.1.

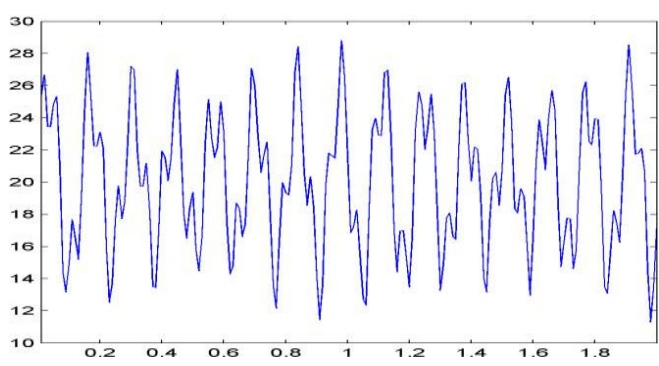

Figure. 1. The profile of wind input speed of 17 w32 m/s

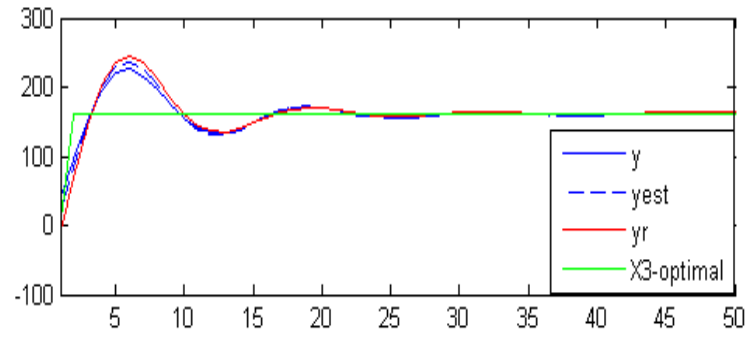

Figure. 2. Rotor speed (rd/s) with Ho observer based

The above figure is presenting the speed rotor tracking. It is clear that the curve follws its reference for the different wind speed variations. Thus, we can deduce that the designed T-S fuzzy controller ensures a good tracking performance. The following figure shows the membership function .

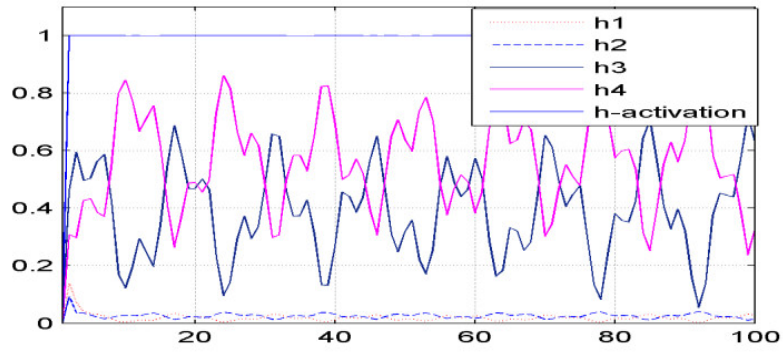

Figure. 3.membership function

\section{Conclusion}

In the present paper, we have proposed a sufficient condition for robust stabilization of uncertain non linear system with external disturbances .a robust fuzzy tracking controller and robust fuzzy observer are devllepoed and analyzed. The main result of this work is the quasi-LMI formulation that can be applied for control design of uncertain and disturbed wind turbine. Based on the T-S fuzzy model, a fuzzy observer based fuzzy controller is developed to reduce the tracking error as much as possible by minimizing the effect of the disturbance level.

\section{REFERENCES}

[1] (Takagi \& Sugeno, 1985) Takagi, T., and Sugeno, M., Fuzzy identification of systems and its application to modelling and control". IEEE Trans. Syst., Man and Cyber, Vol.1115, pp. 116-132, 1985

[2] ShaochengTong,TaoWang,Han-Xiong Li "Fuzzy robust tracking control for uncertain nonlinear systems " (2002)

[3] K. Rai Lee, E. Tae Jeung, H. Bae Park"Robust fuzzy Hocontrol for uncertain nonlinear systems via state feedback: an LMI approach"(2001) 
Kaoutar Lahmadi, Ismail Boumhidi; Fuzzy Robust Hoo Tracking Control For Wind Generator System: LMI approach. Transactions on Machine Learning and Artificial Intelligence, Vol 5 No 4 August (2017); pp: 43-54

[4] B.Mansouri, N. Manamanni, A.Hamzaoui and J. Zaytoon "Tracking control for uncertain Takagi Sugeno fuzzy systems with external disturbances"(2005)

[5] S. Bououden, M. Chadli,, S. Filali, A. El Hajjaji “Fuzzy model based multivariable predictive control of a variable speed wind turbine: LMI approach"(2012)

[6] K. Tanaka, and K. O. Wang. Fuzzy Control Systems Design and Analysis: A linear Matrix Inequality Approach.John Wiley \& Sons, Inc., 2001

[7] Boyd, S., El Ghaoui, L., Feron, E., \&Balakrishnan, V. (1994). Linearmatrix inequalities in system and control theory. In SIAM Studies in Applied Mathematics: vol. 15. Philadelphia, PA: Society for Industrial and Applied Mathematics (SIAM)

[8] B. Mansouri, N. Manamanni, K. Guelton, A. Kruszewski, T.M. Guerra “Output feedback LMI tracking control conditions withH1criterion for uncertain and disturbed T-S models"(2008)

[9] N. Harrabi, M. Kharrat, M. Souissi, A. Aitouche "Maximum Power Point Tracking of a Wind Generation System Based on T-S Fuzzy Model"(2015)

[10] C.Tseng, B.Chen, and H.Uang “Fuzzy Tracking Control Design for Nonlinear Dynamic Systems via T-S Fuzzy Model"(2001)

[11] D.Zhang, Q. Han and X. Jia "Tracking Control for Network-Based T-S Fuzzy Systems With Asynchronous Constraints"(2012)

[12] S. Bezzaoucha, B. Marx, D. Maquin, J. Ragot “Model Reference Tracking Control for Nonlinear Systems described by Takagi-Sugeno Structure"(2013)

[13] K. Ben Meziane, I. Boumhidi “An Interval Type-2 Fuzzy Logic PSS with the optimal H $\infty$ tracking control for multi-machine power system"(2015)

[14] A. Abdelkrim, C. Ghorbel, M. Benrejeb “LMI-based tracking control for Takagi-Sugeno fuzzy model”(2010)

[15] F.Khaber, A.Hamzaoui, K. Zehar "State Feedback Controller Design via Takagi-Sugeno fuzzy Model : A Linear Matrix Inequalities Approach"

[16] J. Zhang, M.Fei, T. Yang and Y. Tan "Robust Fuzzy Tracking Control of Nonlinear Systems with Uncertainty Via T-S Fuzzy Model"(2006)

[17] Allouche,,M. Chaabane,M. Souissi,D. Mehdi ,F. Tadeo "STATE FEEDBACK TRACKING CONTROL FOR INDIRECT FIELD-ORIENTED INDUCTION MOTOR USING FUZZY APPROACH”(2013

[18] Chang-Hua Lien“An efficient method to design robust observer-based control of uncertain linear systems "(2004)

[19] M. Chadli and A. El Hajjaji “Wind energy conversion systems control using T-S fuzzy modeling"(2010 